\title{
Long noncoding RNA SLC9A3-AS1 increases E2F6 expression by sponging microRNA-486-5p and thus facilitates the oncogenesis of nasopharyngeal carcinoma
}

\author{
JIANSHENG LI, DONGZHI LI, XIANHUA ZHANG, CUIJUAN LI and FENGJUAN ZHU
}

Department of Otolaryngology, The People's Hospital of Rizhao, Rizhao, Shandong 276826, P.R. China

Received February 7, 2021; Accepted April 19, 2021

DOI: $10.3892 /$ or.2021.8116

\begin{abstract}
Long noncoding RNA SLC9A3 antisense RNA 1 (SLC9A3-AS1) plays a central role in lung cancer; yet, its functions in nasopharyngeal carcinoma (NPC) have not been elucidated. The present study revealed the roles of SLC9A3-AS1 in NPC and dissected the mechanisms downstream of SLC9A3-AS1. SLC9A3-AS1 levels in NPC were assessed by applying RT-qPCR. The modulatory role of SLC9A3-AS1 interference on NPC cells was examined using numerous functional experiments. High expression of SLC9A3-AS1 was observed in NPC samples. Patients with NPC with a high level of SLC9A3-AS1 experienced a shorter overall survival than those with a low SLC9A3-AS1 level. Loss of SLC9A3-AS1 reduced NPC cell proliferation, colony formation, migration, and invasion but induced cell apoptosis in vitro. Animal experiments further revealed that the depletion of SLC9A3-AS1 hindered NPC tumour growth in vivo. As a competitive endogenous RNA, SLC9A3-AS1 sponged microRNA-486-5p (miR-486-5p), consequently upregulating E2F transcription factor 6 (E2F6). Finally, the effects of SLC9A3-AS1 silencing on NPC cells were reversed by inhibiting miR-486-5p or overexpressing E2F6. In summary, SLC9A3-AS1 exerted carcinogenic effects on NPC cells by adjusting the miR-486-5p/E2F6 axis. Accordingly, the newly identified SLC9A3-AS1/miR-486-5p/E2F6 pathway may offer attractive therapeutic targets for future development.
\end{abstract}

\section{Introduction}

Nasopharyngeal carcinoma (NPC), originating from the nasopharyngeal epithelium, is the most prevalent type of head and

Correspondence to: Professor Fengjuan Zhu, Department of Otolaryngology, The People's Hospital of Rizhao, 126 Taian Road, Rizhao, Shandong 276826, P.R. China

E-mail: zfj18263372760@126.com

Key words: nasopharyngeal carcinoma, long noncoding RNA, microRNA, ceRNA, SLC9A3 antisense RNA 1, E2F transcription factor 6 neck cancer in Southeast Asian populations (1). It is characterized by local aggressiveness and early distant metastasis (2). In China, approximately 60,600 new NPC cases are diagnosed every year, accounting for $40 \%$ of the global cases (3). Currently, intensity-modulated radiation therapy combined with neoadjuvant chemotherapy is the primary therapeutic strategy for NPC (4). Efforts focused on cancer diagnosis and treatment have significantly improved the long-term clinical outcomes of patients with early-stage NPC. However, numerous patients are diagnosed with NPC at an advanced stage and have a poor prognosis (5). Local or regional relapse and metastasis are the most common reasons for the management failure (6). Although the mechanisms of NPC oncogenesis and progression are complicated, they are mainly attributed to genetics, viral infections, and environmental factors (7). However, the detailed underlying mechanisms remain poorly understood. Therefore, clarification of the mechanisms of NPC pathogenesis is urgent and necessary for identifying effective anticancer therapies.

Long noncoding RNAs (lncRNAs), whose length is more than 200 nucleotides, are emerging as cancer drug targets (8). They belong to a subset of noncoding RNA molecules that are not translated into proteins but modulate gene expression (9). Formerly, lncRNAs were considered transcriptional 'noise' or cloning artefacts (10). However, they have recently been revealed to be significant regulators of various physical and pathological phenotypes (11). The dysregulation of lncRNAs is closely associated with various processes in NPC oncogenesis and progression $(12,13)$. Numerous lncRNAs are dysregulated in NPC and exert promoting or inhibitory effects on cancer regulation $(14,15)$.

MicroRNAs (miRNAs) are a group of short noncoding transcripts with a length of 17-21 nucleotides (16). They are confirmed as vital gene modulators and work through complementary pairing with the 3'-untranslated regions (UTRs) of their target genes, ultimately triggering mRNA degradation or reducing translation (17). Numerous miRNAs are abnormally expressed in NPC and are essential in inducing malignancy and tumorigenesis (18-20). The theory of competitive endogenous RNA (ceRNA) proposed by Salmena et al (21) has accelerated research on lncRNA mechanisms. LncRNAs function as sponges for miRNA, subsequently abating the inhibitory effects of miRNAs on their targeted mRNAs (21). 
According to a previous study, SLC9A3 antisense RNA 1 (SLC9A3-AS1) was revealed to play a central role in lung cancer (22). However, researchers have not clearly determined whether SLC9A3-AS1 exhibits clinical value in NPC and how it performs its specific functions in NPC. Its underlying mechanisms require further study. Thus, SLC9A3-AS1 was selected as the focus of the present study. SLC9A3-AS1 expression in NPC was analysed, and roles of SLC9A3-AS1 in NPC were investigated. Furthermore, mechanistic experiments were used to dissect the downstream mechanisms of SLC9A3-AS1 in NPC.

\section{Materials and methods}

Patient tissues. The present study was approved by the Ethics Committee of the People's Hospital of Rizhao (Rizhao, China). The experimental procedures were performed in strict compliance with the Declaration of Helsinki (2013 version). In addition, all participants provided written informed consent. A total of 46 NPC tissues were acquired from patients ( 28 males and 18 females; age range, 32-65 years) at our hospital (People's Hospital of Rizhao) between May 2014 to December 2015. The inclusion criteria of NPC were as follows: i) Diagnosed as NPC; ii) had not been treated with preoperative anticancer treatments and iii) agreed to participate in the study. In addition, 15 normal nasopharyngeal epithelial tissues were provided by healthy volunteers ( 9 males and 6 females; age range, 28-57 years) at our hospital. The inclusion criteria of the healthy volunteers were as follows: i) Have not been diagnosed as NPC; and ii) agreed to participate in the study. Patients with other types of human malignancies and patients who had undergone any form of anticancer therapy were excluded from this study. All the excised tissues were immediately flash-frozen and maintained in liquid nitrogen.

Cell lines. The normal nasopharyngeal epithelial cell line NP69 and three NPC cell lines (SUNE1, C666-1 and 6-10B) were purchased from the Laboratory of Cell Biology, Modern Analysis and Testing Center, Central South University (Changsa, China). NP69 cells were grown in keratinocyte serum-free medium (Gibco; Thermo Fisher Scientific, Inc.) containing $30 \mu \mathrm{g} / \mathrm{ml}$ bovine pituitary extract (BD Biosciences). NPC cells were cultured in RPMI-1640 medium supplemented with $10 \%$ fetal bovine serum (FBS), and $1 \%$ penicillin/streptomycin mixture (Gibco; Thermo Fisher Scientific, Inc.). All the cells were grown in saturated humidity with $5 \% \mathrm{CO}_{2}$ at $37^{\circ} \mathrm{C}$.

Cell transfection. Small interfering RNAs (siRNAs) synthesized against SLC9A3-AS1 (si-SLC9A3-AS1) and a corresponding negative control (NC) siRNA (si-NC) were obtained from Shanghai GenePharma Co., Ltd. The si-SLC9A3-AS1\#1 sequence was 5'-CACATGTTTTTTATA ATAAAACA-3'; the si-SLC9A3-AS1\#2 sequence was 5'-ATG TTTTTTATAATAAAACATAG-3'; and the si-NC sequence was 5'-CACGATAAGACAATGTATTT-3'. The miR-486-5p mimic, miR-486-5p inhibitor, and the matching controls (NC mimic and NC inhibitor, respectively) were prepared by Guangzhou RiboBio Co., Ltd. The miR-486-5p mimic sequence was 5'-GAGCCCCGUCGAGUCAUGUCCU-3' and the NC mimic sequence was 5'-UUGUACUACACAAAA GUACUG-3'. The miR-486-5p inhibitor sequence was 5'-CUC GGGGCAGCUCAGUACAGGA-3' and the NC inhibitor sequence was 5'-ACUACUGAGUGACAGUAGA-3'. The pcDNA3.1-E2F6 plasmid was constructed by cloning the E2F6 cDNA into pcDNA3.1 (GenScript Biotech Corporation). The transfection of siRNA (100 pmol), miRNA oligonucleotides (100 pmol) or plasmids ( $4 \mu \mathrm{g}$ ) was performed at room temperature with Lipofectamine ${ }^{\circledR} 2000$ (Invitrogen; Thermo Fisher Scientific, Inc.). At $24 \mathrm{~h}$ post-transfection, Cell Counting Kit-8 (CCK-8), colony-forming and cell migration and invasion assays were performed. Reverse transcription-quantitative polymerase chain reaction (RT-qPCR), flow cytometry and western blotting were carried out after $48 \mathrm{~h}$ of culture.

$R T$ - $q P C R$. A mirVana ${ }^{\mathrm{TM}}$ miRNA Isolation kit (Ambion; Thermo Fisher Scientific, Inc.) was employed for isolating small RNA, which was then reverse transcribed into complementary DNA by applying miRcute miRNA First-Strand cDNA Synthesis Kit (Tiangen Biotech Co., Ltd.) according to the manufacturer's instructions. The expression of miR-486-5p was detected using PCR amplification with a miRcute miRNA qPCR Detection Kit SYBR-Green (Tiangen Biotech Co., Ltd.) according to the manufacturer's instructions, with small nuclear RNA U6 as an internal reference. The thermocycling conditions were as follows: $95^{\circ} \mathrm{C}$ for $15 \mathrm{~min}$; and $94^{\circ} \mathrm{C}$ for $20 \mathrm{sec}$ and $60^{\circ} \mathrm{C}$ for $34 \mathrm{sec}$, for 45 cycles. The extraction of total RNA was performed using TRIzol ${ }^{\circledR}$ (Invitrogen; Thermo Fisher Scientific, Inc.). For SLC9A3-AS1 and E2F6 quantification, reverse transcription was conducted using a PrimeScript reagent Kit with gDNA Eraser (Takara Biotechnology Co., Ltd.). Then, complementary DNA was subjected to quantitative PCR by using PrimeScript ${ }^{\mathrm{TM}}$ RT Master Mix (Takara Biotechnology Co., Ltd.). The thermocycling conditions were as follows: $95^{\circ} \mathrm{C}$ for $30 \mathrm{sec}, 95^{\circ} \mathrm{C}$ for $3 \mathrm{sec}$ and $60^{\circ} \mathrm{C}$ for $30 \mathrm{sec}$, for 40 cycles; $95^{\circ} \mathrm{C}$ for $15 \mathrm{sec}, 60^{\circ} \mathrm{C}$ for $60 \mathrm{sec}$ and $95^{\circ} \mathrm{C}$ for $15 \mathrm{sec}$. SLC9A3-AS1 and E2F6 levels were normalized to GAPDH. All gene expression levels were analysed with the $2^{-\Delta \Delta \mathrm{Ca}}$ method (23).

The primers were designed as follows: SLC9A3-AS1, 5'-CGAGAGAGGGCAGCGGCTAGT-3'(forward) and 5'-TAACTTTCCAAGGCACCCAGCA-3' (reverse); E2F6, 5'-TGTGTCCATGAGAAAAGCTCTAAAA-3' (forward) and 5'-ACCTTGTTTAAGTCAAGAATACCCC-3' (reverse); GAPDH, 5'-ACCTGACCTGCCGTCTAGAAAA-3' (forward) and 5'-TTGAAGTCAGAGGAGACCACCTG-3' (reverse); miR-486-5p, 5'-TCGGCAGGUCCUGUACUGAG-3' (forward) and 5'-CACTCAACTGGTGTCGTGGA-3' (reverse); U6, 5'-CTCGCTTCGGCAGCACA-3' (forward) and 5'-AACGCT TCACGAATTTGCGT-3' (reverse).

CCK-8 assay. The transfected NPC cells were harvested and counted separately after being inoculated in 96-well plates. Each well covered $100 \mu \mathrm{l}$ cell suspension harbouring 3,000 cells. Cells were cultured in saturated humidity with $5 \% \mathrm{CO}_{2}$ at $37^{\circ} \mathrm{C}$. For cell proliferation measurement, $10 \mu \mathrm{l}$ of CCK-8 solution (Beyotime Institute of Biotechnology) were added and incubated at $37^{\circ} \mathrm{C}$ for $2 \mathrm{~h}$. Subsequently, the cells were counted at $450 \mathrm{~nm}$ with an ELISA plate reader. The assay 
was executed every $24 \mathrm{~h}$ until $72 \mathrm{~h}$, and acquired data was applied for plotting a growth curve.

Colony-forming assay. The transfected NPC cells were harvested and counted separately. Then, $2 \mathrm{ml}$ of the cell suspension containing 1,000 cells per well was seeded on 6 -well plates. After 2 weeks of incubation at $37^{\circ} \mathrm{C}$, the cells were washed with phosphate-buffered saline (PBS), and then they were fixed using $4 \%$ paraformaldehyde at room temperature for $30 \mathrm{~min}$ and stained using $0.5 \%$ crystal violet at room temperature for $30 \mathrm{~min}$. Ultimately, the formed colonies (>50 cells) were counted under an inverted light microscope (Leica Microsystems $\mathrm{GmbH}$ ).

Flow cytometry. Transfected cells were detached with EDTA-free trypsin and centrifuged at room temperature with $1,000 \mathrm{x} g$ for $5 \mathrm{~min}$. The percentage of apoptotic cells was determined employing eBioscience ${ }^{\mathrm{TM}}$ Annexin V Apoptosis Detection Kit FITC (Invitrogen; Thermo Fisher Scientific, Inc.) according to the manufacturer's instructions. After one rinse with PBS and one rinse with $1 \mathrm{X}$ binding buffer, the cells were collected and resuspended in $100 \mu \mathrm{l}$ of $1 \mathrm{X}$ binding buffer. Then, fluorochrome-conjugated Annexin V (5 $\mu \mathrm{l})$ and propidium iodide $(5 \mu \mathrm{l})$ was appended to the cell suspension and cultured for $20 \mathrm{~min}$ at $20-25^{\circ} \mathrm{C}$ under darkness. Finally, the cell apoptosis rate was assayed with a FACSCalibur flow cytometer (BD Biosciences). CellQuest software (version 2.9; BD Biosciences) was utilized for data abalysis.

Cell migration and invasion assays. Transwell chambers ( $8 \mu \mathrm{m}$; Corning Inc.) were utilized to uncover the cell migratory and invasive capacities. For the determination of cell migration, $200 \mu \mathrm{l}$ of FBS-free medium carrying $5 \times 10^{4}$ cells were seeded to the upper chambers. In the lower chambers, $600 \mu \mathrm{l}$ of RPMI-1640 medium mixed with $20 \%$ FBS was plated and used as the chemoattractant. Subsequent to 24-h culture, the non-migrated cells were gently removed from the upper chamber, while the migrated cells were fixed with $4 \%$ paraformaldehyde at room temperature for $30 \mathrm{~min}$, stained with $0.5 \%$ crystal violet at room temperature for $30 \mathrm{~min}$, and observed and counted under an inverted light microscope (x200, magnification; Leica Microsystems $\mathrm{GmbH}$ ). The invasion test used Matrigel (Corning Inc.) precoated-Transwell chambers and the same subsequent experimental procedure as the migration assay.

Lentivirus production and infection. Lentiviruses were produced using a second-generation lentiviral system. The short hairpin RNA (shRNA) for SLC9A3-AS1 (sh-SLC9A3-AS1) and NC shRNA (sh-NC; Shanghai GenePharma Co., Ltd.) were cloned into the pLKO.1 plasmid. The sh-SLC9A3-AS1 sequence was 5'-CCGGATGTTTTT TATAATAAAACATAGCTCGAGCTATGTTTTATTATA AAAAACATTTTTTG-3' and sh-NC sequence was 5'-CCG GCACGATAAGACAATGTATTTCTCGAGAAATACATT GTCTTATCGTGTTTTTG-3'. The constructed plasmids were transfected into $293 \mathrm{~T}$ cells (National Collection of Authenticated Cell Cultures, Shanghai, China) with the psPAX2 packaging plasmid and pMD2. G envelope plasmid to generate lentiviruses. The ratio of lentiviral plasmid: psPAX2: pMD2.G: pLKO.1 was 1:1:2, and a total of $30 \mu \mathrm{g}$ plasmids were used for lentivirus package. After $48 \mathrm{~h}$ of incubation at $37^{\circ} \mathrm{C}$, lentiviruses overexpressing sh-SLC9A3-AS1 or sh-NC were collected and mixed with polybrene $(5 \mu \mathrm{g} / \mathrm{ml}$; Sigma-Aldrich; Merck KGaA) and culture medium. Then, the mixture was added into SUNE1 cells with an MOI=5 for lentivirus infection. The infected cells were treated at $37^{\circ} \mathrm{C}$ with puromycin $(2 \mu \mathrm{g} / \mathrm{ml}$; Sigma-Aldrich) for 10 days to obtain SUNE1 cells with stable SLC9A3-AS1 depletion. The maintenance concentration was $0.3 \mu \mathrm{g} / \mathrm{ml}$.

Animal studies. All experiments involving animals were approved by the Animal Ethics Committee of The People's Hospital of Rizhao. A total of 6 male BALB/c nude mice aged 4-6 weeks (weight, 20 g; Hunan SJA Laboratory Animal Co., Ltd.) received a subcutaneous injection of SUNE1 cells with stable SLC9A3-AS1 knockdown to generate tumours. All mice were housed under specific pathogen-free conditions at $25^{\circ} \mathrm{C}$ and 50\% humidity, with a 10:14 light/dark cycle and ad libitum access to food and water. The tumours were measured with Vernier callipers every 5 days to calculate the tumour volume using the following formula: Tumour volume $=0.5 \mathrm{x}$ length $\mathrm{x}$ width ${ }^{2}$. On day 35 , the mice were euthanized by cervical dislocation, and the tumour xenografts were processed for weighing, RT-qPCR and western blotting.

Immunohistochemistry. Tumor xenografts were fixed in $4 \%$ neutral formalin at room temperature for $48 \mathrm{~h}$, soaked in $4 \%$ paraffin, and were cut into $4-\mu \mathrm{m}$-thick sections. Xylene was applied for deparaffinising. Next, rehydration was implemented with an ethanol gradient. After culture with $0.3 \% \mathrm{H}_{2} \mathrm{O}_{2}$ for $30 \mathrm{~min}$ and blocking with $5 \%$ bovine serum albumin (R\&D Systems) for $45 \mathrm{~min}$ at $37^{\circ} \mathrm{C}$, the slides were incubated all night with E2F6 (product code ab53061; 1:1,000 dilution) or Ki-67 (product code ab15580; 1:1,000 dilution; both from Abcam) at $4^{\circ} \mathrm{C}$, followed by $45 \mathrm{~min}$ of treatment at room temperature with a horseradish peroxidase-conjugated secondary antibody (cat. no. ab205718; Abcam; 1:500 dilution). Then, 3,3'-diaminobenzidine (DAB) color reagent was applied to detect the antibody binding, and tumor xenografts were counterstained with $1 \%$ hematoxylin at room temperature for $10 \mathrm{~min}$ and dehydrated in ethanol. Finally, image acquisition was implemented utilizing a light microscope (x200, magnification).

Subcellular fractionation assay. The nuclear and cytoplasmic fractions of NPC cells were separated using a PARIS kit (Thermo Fisher Scientific, Inc.), according to the manufacturer's instructions. Next, total RNA was isolated from each fraction for RT-qPCR to quantify SLC9A3-AS1 in the nuclear and cytoplasmic fractions.

Bioinformatics analysis. The binding between SLC9A3-AS1 and miR-486-5p was predicted applying ENCORI (starBase 3.0; http://starbase.sysu.edu.cn/) and miRDB (http:// www.mirdb.org/). Two bioinformatics tools, ENCORI and TargetScan version 7.2 (http://www.targetscan.org), were adopted to identify the potential targets of miR-486-5p.

Luciferase reporter assay. The SLC9A3-AS1 and E2F6 fragments harbouring the predicted wild-type (WT) binding 

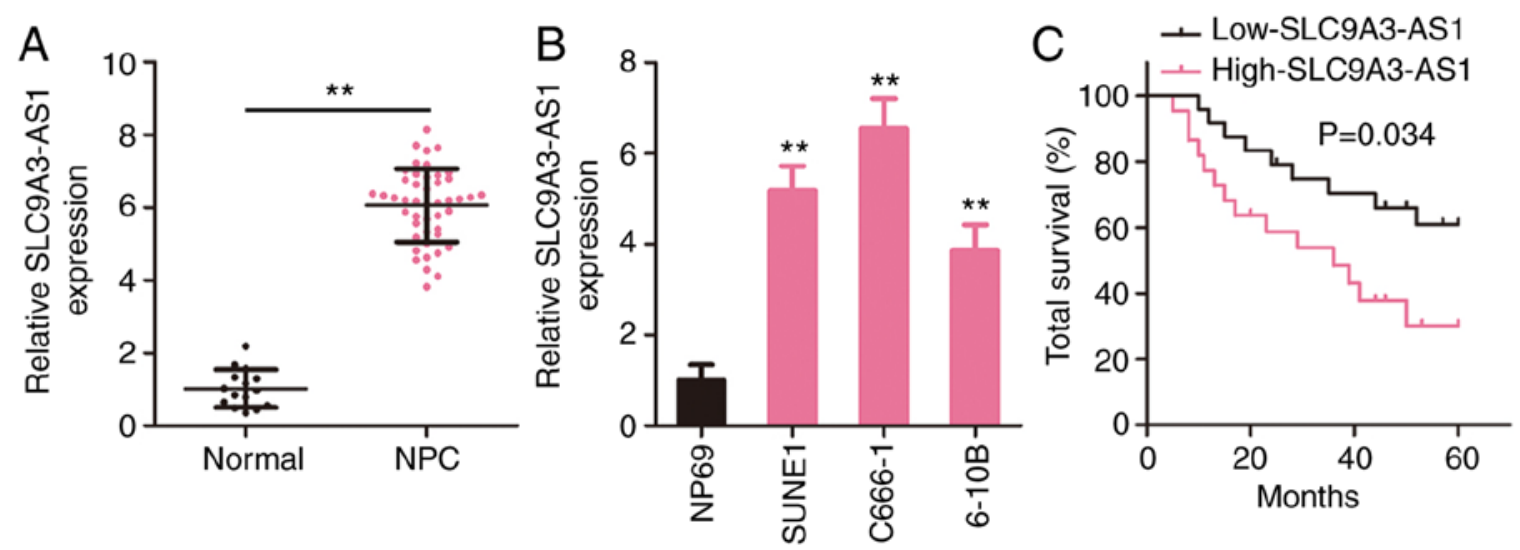

Figure 1. SLC9A3-AS1 is overexpressed in NPC and negatively associated with patient prognosis. (A) SLC9A3-AS1 expression in NPC tissues was detected using RT-qPCR. (B) SLC9A3-AS1 level in NPC cell lines was assayed using RT-qPCR. (C) The relationship between SLC9A3-AS1 level and overall survival in NPC patients. ${ }^{* *} \mathrm{P}<0.01$. SLC9A3-AS1, SLC9A3 antisense RNA 1; NPC, nasopharyngeal carcinoma; RT-qPCR, reverse transcription-quantitative polymerase chain reaction.

site (Shanghai GenePharma Co., Ltd.) were cloned into the pmirGLO luciferase plasmid (Promega Corporation) to create the reporter plasmids WT-SLC9A3-AS1 and WT-E2F6. Mutant (MUT) reporter plasmids, MUT-SLC9A3-AS1 and MUT-E2F6, were also constructed. NPC cells were seeded into 24-well plates and co-transfected with the reporter plasmids alongside miR-486-5p mimic or NC mimic applying Lipofectamine $^{\circledR} 2000$ (Invitrogen; Thermo Fisher Scientific, Inc.). Subsequently, $48 \mathrm{~h}$ later, the transfected cells were lysed to detect their luciferase activity employing a dual-luciferase reporter assay system (Promega Corporation). Renilla luciferase activity was applied to normalize the firefly luciferase activity.

RNA immunoprecipitation (RIP). RIP was implemented with an EZ-Magna RIP ${ }^{\mathrm{TM}}$ RNA-Binding Protein Immunoprecipitation Kit (cat. no. 03-110; EMD Millipore) according to the manufacturer's instructions. NPC cells were lysed with RIP lysis buffer. After centrifugation at $1,000 \times \mathrm{g}$ for $10 \mathrm{~min}$ at $4^{\circ} \mathrm{C}$, $10 \%$ of the cell lysate was regarded as the positive control (input). The remaining lysate was cultured at $4^{\circ} \mathrm{C}$ overnight with magnetic beads, which were already conjugated with an anti-Argonaute 2 (Ago2) or IgG antibody (negative control) (both from cat. no. 03-110; dilution, 1:5,000; EMD Millipore). Subsequently, the immunoprecipitated RNA was purified and analysed using RT-qPCR.

Western blotting. Total protein was extracted from the cells lysed with RIPA lysis buffer supplemented with phenylmethylsulfonyl fluoride (both from Sangon Biotech Co., Ltd.). The quantification of total protein was conducted with a BCA Protein Assay Kit (Sangon Biotech Co., Ltd.). Equal amounts of proteins $(20 \mu \mathrm{g})$ were separated by $10 \%$ SDS-PAGE gels, followed by transferring onto PVDF membranes. After being blocked with $5 \%$ fat-free milk at room temperature for $2 \mathrm{~h}$, the blots were probed with primary antibodies targeting E2F6 (product code ab155978; 1:1,000 dilution) or GAPDH (product code ab181602; 1:1,000 dilution; both from Abcam) overnight at $4^{\circ} \mathrm{C}$. The blots were treated with a horseradish peroxidase-conjugated secondary antibody (product code ab205718; 1:5,000 dilution; Abcam) at room temperature for $2 \mathrm{~h}$ and then visualized with the Immobilon ECL Ultra Western HRP Substrate (Merck Millipore; Merck KGaA).

Statistical analysis. The results from three independent experiments were expressed as the means \pm standard deviations. Multigroup comparisons were conducted applying one-way analysis of variance (ANOVA), and Tukey's test was used for post hoc comparisons. Both paired and unpaired Student's t-tests were used to contrast the differences between the two groups. The survival of patients with NPC was examined using Kaplan-Meier method, and the overall survival curves were compared using the log-rank test. Pearson's correlation analysis was carried out to assess correlations between the expression of various genes. $\mathrm{P}<0.05$ was considered to indicate a statistically significant difference.

\section{Results}

SLC9A3-AS1 is upregulated in NPC and correlated with apoor clinical outcome. To investigate the pattern of SLC9A3-AS1 expression in NPC, SLC9A3-AS1 level in 46 NPC tissues and 15 normal nasopharyngeal epithelial tissues was measured utilizing RT-qPCR. Compared with normal nasopharyngeal epithelial tissues, SLC9A3-AS1 was expressed at a higher level in NPC tissues (Fig. 1A). Additionally, all three NPC cell lines exhibited higher SLC9A3-AS1 expression compared with NP69 (Fig. 1B). In addition, all patients with NPC were assigned to a low-SLC9A3-AS1 $(n=23)$ or high-SLC9A3-AS1 $(n=23)$ group according to the median value of SLC9A3-AS1. The overall survival in the low-SLC9A3-AS1 group was significantly increased compared with that in the high-SLC9A3-AS1 group (Fig. 1C). Based on these data, SLC9A3-AS1 may participate in the genesis and development of NPC.

Inhibition of SLC9A3-AS1 supresses the malignant processes of NPC cells in vitro. Next, the functions of SLC9A3-AS1 in NPC cells were delineated. SUNE1 and C666-1 cells expressed the highest SLC9A3-AS1 expression level among the three NPC cells; therefore, these cell lines were selected for functional experiments. First, SLC9A3-AS1 was depleted in SUNE1 and C666-1 cells by transfecting si-SLC9A3-AS1. 

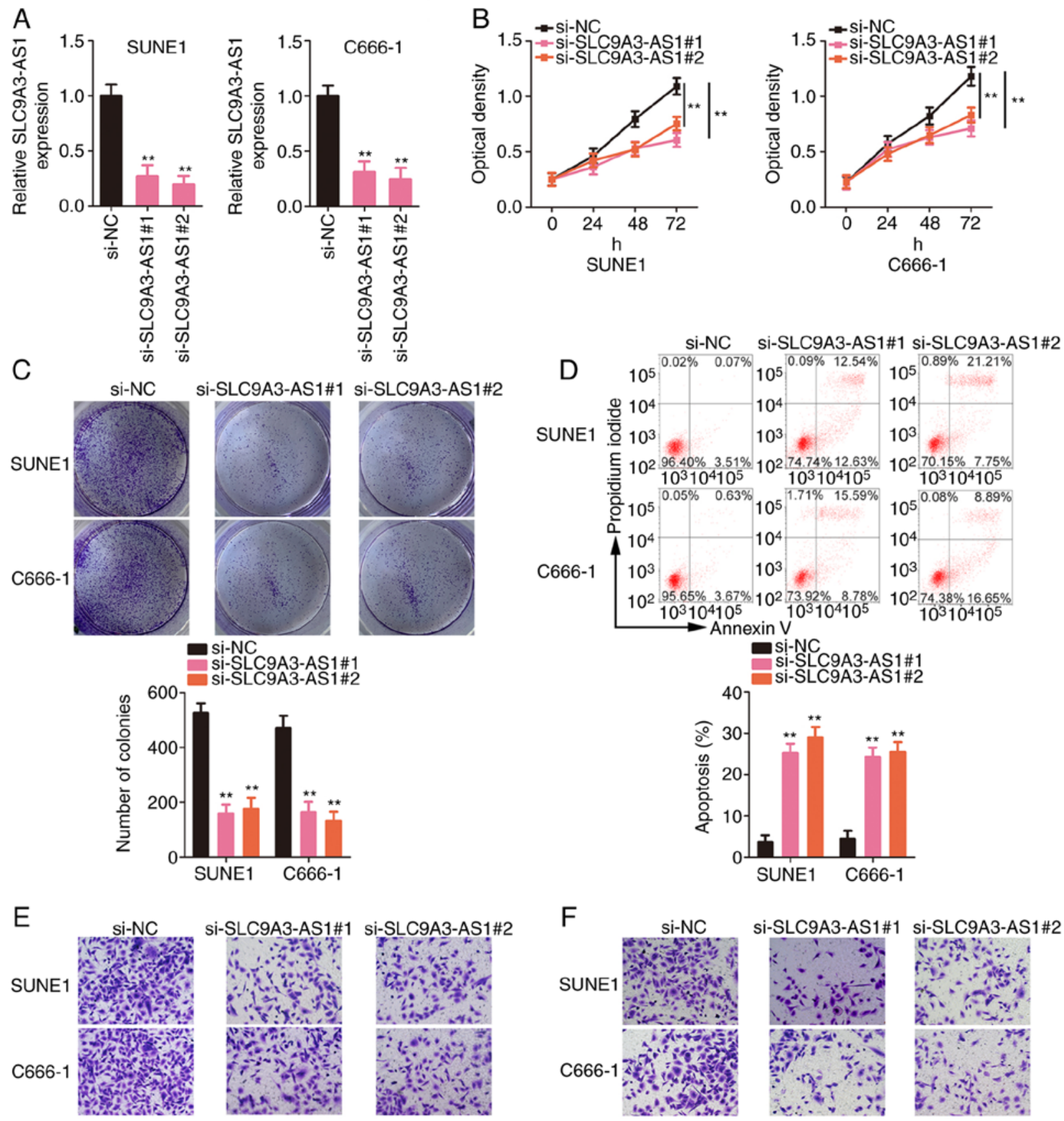

si-SLC9A3-AS1\#1 si-SLC9A3-AS1\#2
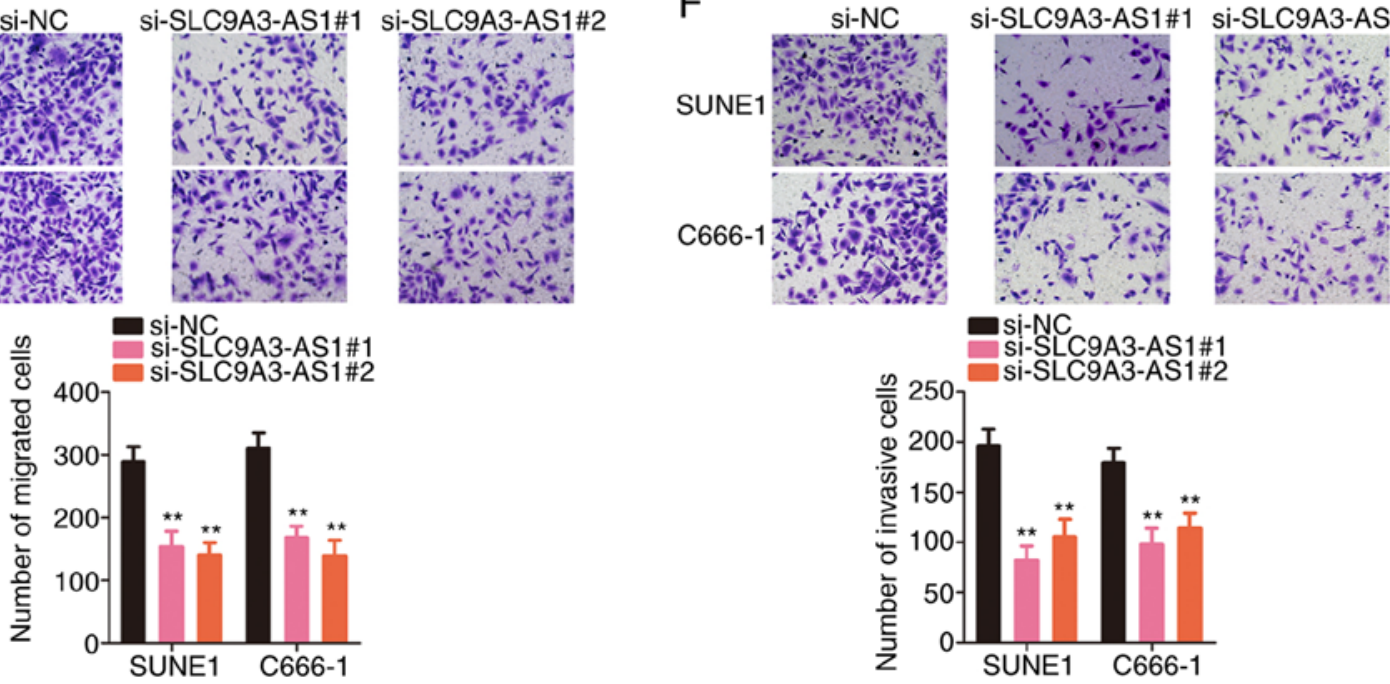

Figure 2. SLC9A3-AS1 depletion suppresses the malignant phenotype of NPC cells. (A) NPC cells were transfected with si-SLC9A3-AS1 or si-NC; the siRNA interference efficiency was determined using reverse transcription-quantitative polymerase chain reaction. (B and C) The effects of SLC9A3-AS1 knockdown on NPC cell proliferation and colony-forming was measured by CCK-8 and colony formation assays, respectively. (D) The apoptosis of NPC cells with SLC9A3-AS1 knockdown was assayed by flow cytometry. (E and F) The migratory and invasive capacities were measured in SLC9A3-AS1 deficient-NPC cells. " P<0.01. SLC9A3-AS1, SLC9A3 antisense RNA 1; NPC, nasopharyngeal carcinoma; si-, small interfering; NC, negative control.

Two siRNAs targeting SLC9A3-AS1 were used to avoid off-target effects; both siRNAs significantly silenced SLC9A3-AS1 expression (Fig. 2A). The proliferative capacity of NPC cells was suppressed after SLC9A3-AS1 depletion (Fig. 2B). Furthermore, transfection with si-SLC9A3-AS1 significantly decreased the formation of NPC cell colonies 
A

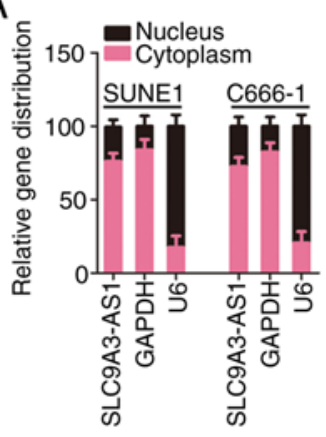

E

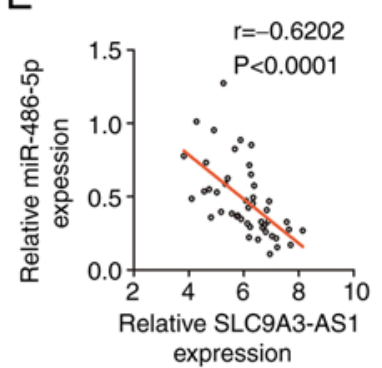

B

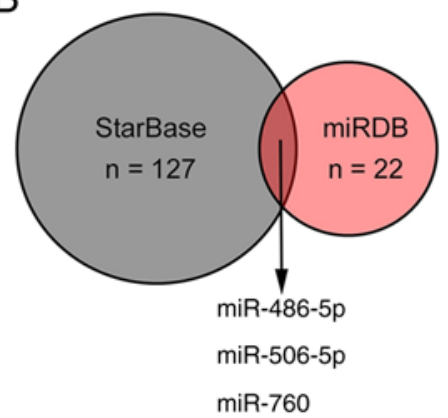

$\mathrm{F}$

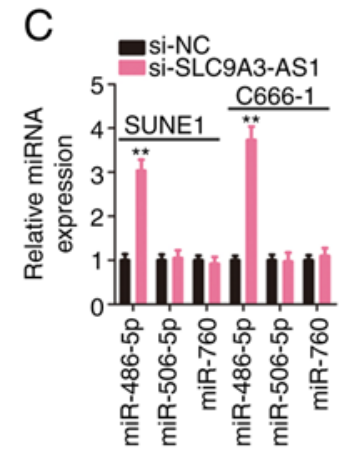

D

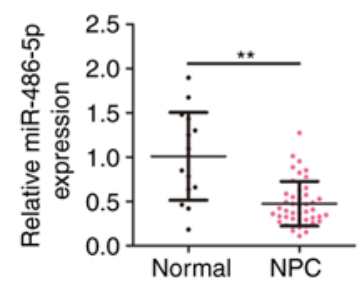

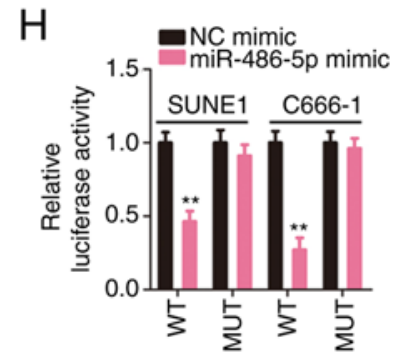
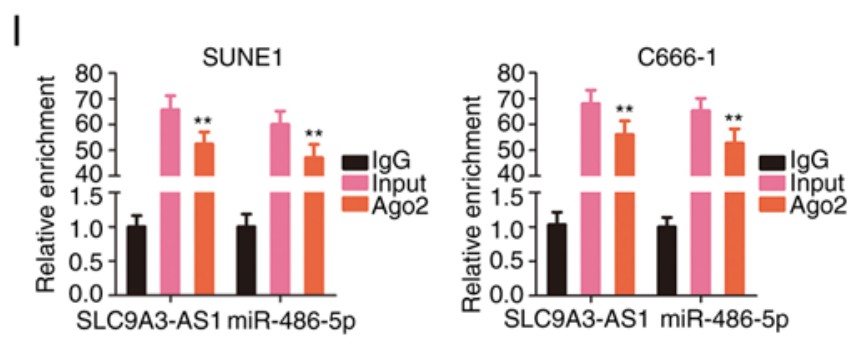

Figure 3. SLC9A3-AS1 sponges miR-486-5p in NPC. (A) The location of SLC9A3-AS1 in SUNE1 and C666-1 cells was confirmed by performing a subcellular fractionation assay. (B) The starBase and miRDB databases were searched for putative target miRNAs of SLC9A3-AS1, and the three intersecting miRNAs are presented. (C) The levels of 3 candidate miRNAs in SLC9A3-AS1-silenced NPC cells were measured using RT-qPCR. (D) miR-486-5p expression level in NPC tissues was determined by applying RT-qPCR. (E) An inverse relationship between miR-486-5p and SLC9A3-AS1. (F) The WT and MUT binding sequences of miR-486-5p within SLC9A3-AS1. (G) RT-qPCR was conducted for the confirmation of miR-486-59 overexpression in NPC cells when miR-486-5p mimic was transfected. (H) The luciferase activity of miR-486-5p overexpressed-NPC cells driven by WT-SLC9A3-AS1 or MUT-SLC9A3-AS1. (I) The enrichment of SLC9A3-AS1 and miR-486-5p in different groups was determined using a modified RIP assay. ${ }^{* *}$ P<0.01. SLC9A3-AS1, SLC9A3 antisense RNA 1; miRNA or miR, microRNA; NPC, nasopharyngeal carcinoma; RT-qPCR, reverse transcription-quantitative polymerase chain reaction; WT, wild-type; MUT, mutant; si-small interfering; NC, negative control.

(Fig. 2C). Additionally, flow cytometric data revealed that the apoptosis of SLC9A3-AS1-deficient NPC cells was significantly increased (Fig. 2D). Furthermore, migration (Fig. 2E) and invasion (Fig. 2F) activities were inhibited in NPC cells with SLC9A3-AS1 knockdown. Thus, SLC9A3-AS1 exerted tumour-promoting effects on NPC cells.

SLC9A3-AS1 directly sponges miR-486-5p in NPC. The mechanism of SLC9A3-AS1 was further dissected by investigating the subcellular distribution of SLC9A3-AS1 in NPC cells. SLC9A3-AS1 was primarily localized in NPC cell cytoplasm (Fig. 3A), suggesting a role for SLC9A3-AS1 as a miRNA sponge. The putative target miRNAs of SLC9A3-AS1 were predicted using starBase and miRDB, and three overlapping miRNAs from the queries were identified (Fig. 3B). The targeting relationships between SLC9A3-AS1 and the miRNAs were examined by assessing the levels of the three overlapping miRNAs in SLC9A3-AS1-silenced NPC cells using RT-qPCR. Loss of SLC9A3-AS1 expression increased miR-486-5p expression but exerted no effect on the levels of miR-506-5p and miR-760 (Fig. 3C). In addition, miR-486-5p was considerably downregulated in NPC tissues (Fig. 3D), and a modest but significant inverse relationship was validated between SLC9A3-AS1 and miR-486-5p expression levels (Fig. 3E). As depicted in Fig. 3F, miR-486-5p contained sequences complementary to SLC9A3-AS1. Next, the target interaction between miR-486-5p and SLC9A3-AS1 in NPC cells was verified employing a luciferase reporter assay. miR-486-5p upregulation (Fig. 3G) induced by the miR-486-5p mimic significantly reduced the luciferase activity driven by WT-SLC9A3-AS1; however, the modulatory effect was abolished when its binding site for SLC9A3-AS1 was mutated (Fig. 3H). Furthermore, the RIP results confirmed that compared with the IgG group, the enrichment of miR-486-5p and SLC9A3-AS1 was increased in the Ago2 group (Fig. 3I). Based on these results, SLC9A3-AS1 was revealed to sponge miR-486-5p in NPC.

E2F6 is a downstream target of miR-486-5p in NPC. The roles of miR-486-5p were studied by overexpressing it in SUNE1 and C666-1 cells and performing gain-of-function 
A
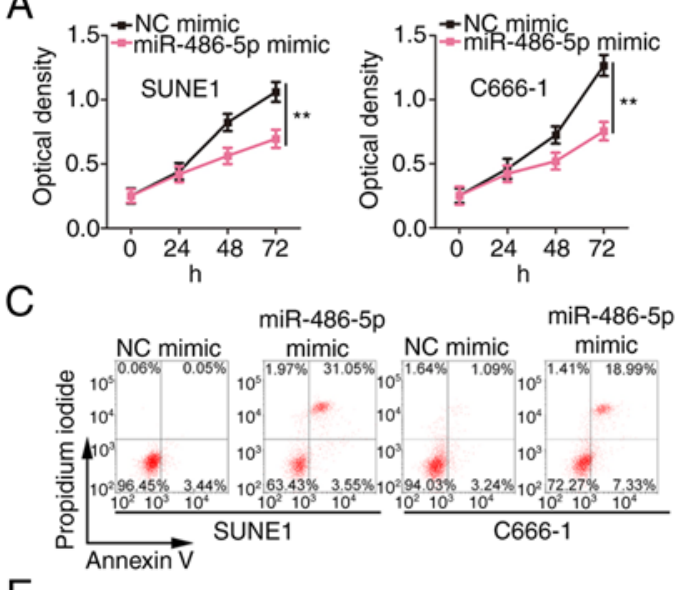

B
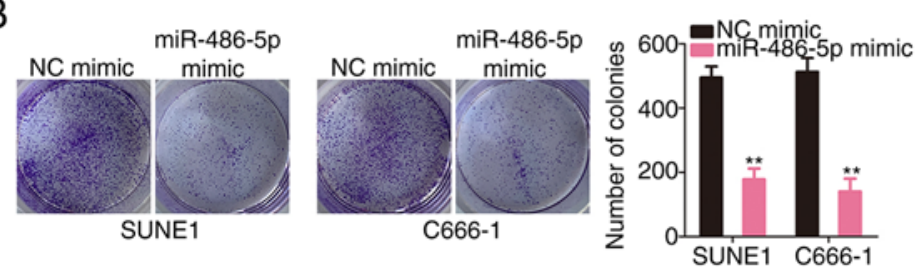

D
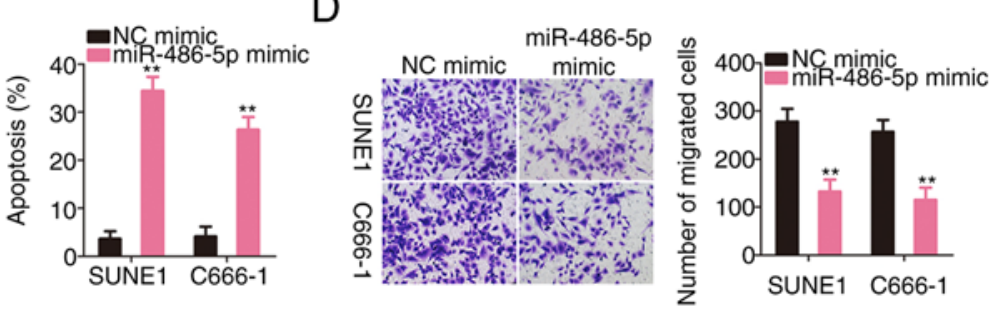
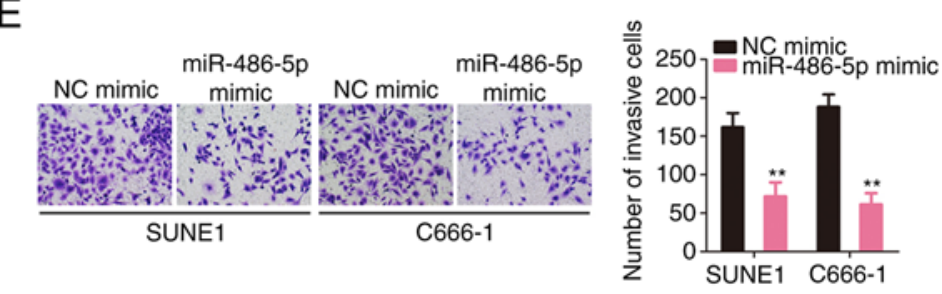

F $\quad$ Target site: Position 1625-1631 of E2F6 3'-UTR

WT-E2F6 5' ...UGACAGAAUAGGGAAUACAGGAG... 3'

miR-486-5p 3' GAGCCCCGUCGAGUCAUUGUCCU 5'

MUT-E2F6 5' ...UGACAGAAUAGGGAACAUGUCCG... 3'

Mutant sequences

G

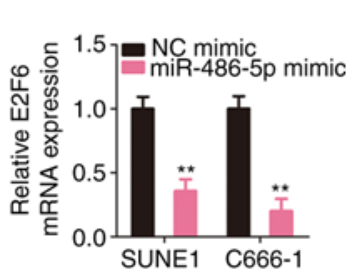

J

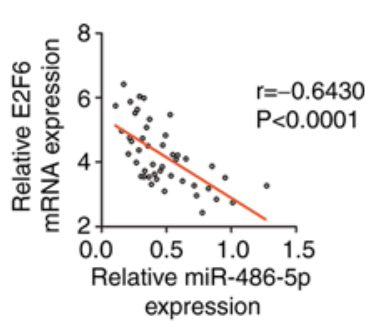

$\mathrm{H}$

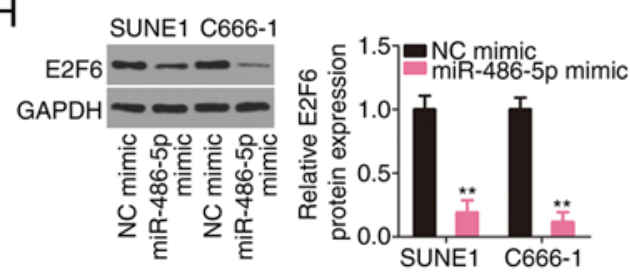

I

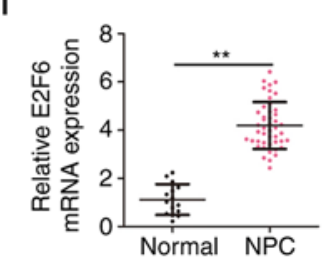

K
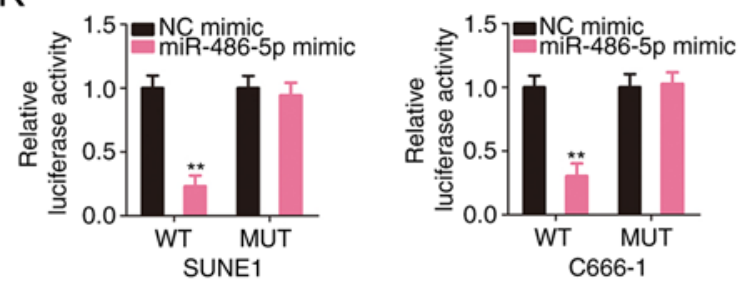

Figure 4. E2F6 is a direct target of miR-486-5p. (A and B) Cell proliferation and colony formation was assessed in miR-486-5p-overexpressing NPC cells. (C) The apoptosis was detected in NPC cells overexpressing miR-486-5p. (D and E) The effects of miR-486-5p overexpression on NPC cell migration and invasion were assessed. (F) The WT and MUT binding sites of miR-486-5p within E2F6 3'-UTR. (G and H) E2F6 levels were measured in NPC cells which were treated with miR-486-5p mimic or NC mimic. (I) Reverse transcription-quantitative polymerase chain reaction was implemented for the measurement of miR-486-5p level in NPC tissues. (J) An inverse relationship between miR-486-5p and E2F6 levels in NPC tissues. (K) NPC cells were co-transfected with WT-E2F6 or MUT-E2F6 and miR-486-5p mimic or NC mimic, and their respective luciferase activities were measured. ${ }^{* *} \mathrm{P}<0.01$. E2F6, E2F transcription factor 6; miR, microRNA; NPC, nasopharyngeal carcinoma; WT, wild-type; MUT, mutant; NC, negative control.

assays. Compared to the NC mimic group, the proliferative (Fig. 4A) and colony-forming (Fig. 4B) capacities of NPC cells were restrained after miR-486-5p overexpression. In addition, exogenous miR-486-5p caused a significant increase in NPC cell apoptosis (Fig. 4C). Moreover, the migration and invasion of NPC cells overexpressing miR-486-5p were supressed (Fig. 4D and E). Then, the mechanisms involving miR-486-5p in NPC cells were studied. According to the bioinformatics prediction, the 3'-UTR of E2F6 contained potential binding sequences for miR-486-5p (Fig. 4F). E2F6 expression levels were reduced in miR-486-5p overexpressed-NPC cells (Fig. 4G and H). The RT-qPCR data revealed the upregulation of E2F6 in NPC tissues (Fig. 4I), which presented a negative relationship with miR-486-5p expression (Fig. 4J).
Furthermore, the luciferase activity driven by WT-E2F6 was downregulated by the miR-486-5p mimic in NPC cells; but, the luciferase activity of MUT-E2F6 was unaltered in response to miR-486-5p reintroduction (Fig. 4K). In summary, miR-486-5p was revealed to directly target E2F6 in NPC.

SLC9A3-AS1 indirectly regulates E2F6 expression in NPC cells by controlling $m i R-486-5 p$. Since the relationships of miR-486-5p with its upstream regulator SLC9A3-AS1 and its downstream target E2F6 were confirmed, the regulatory interactions among the three RNAs were further analysed. E2F6 expression levels (Fig. 5A and B) were significantly suppressed in NPC cells transfected with si-SLC9A3-AS1. Additionally, a positive relationship between SLC9A3-AS1 and E2F6 
A

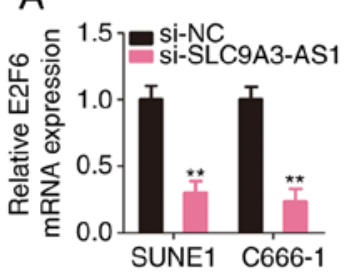

$\mathrm{B}$

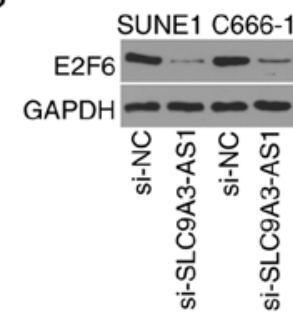

C

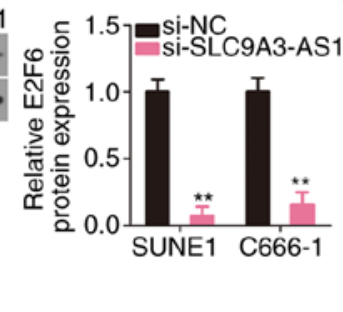

E

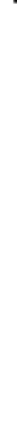

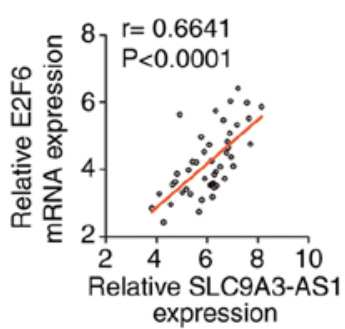
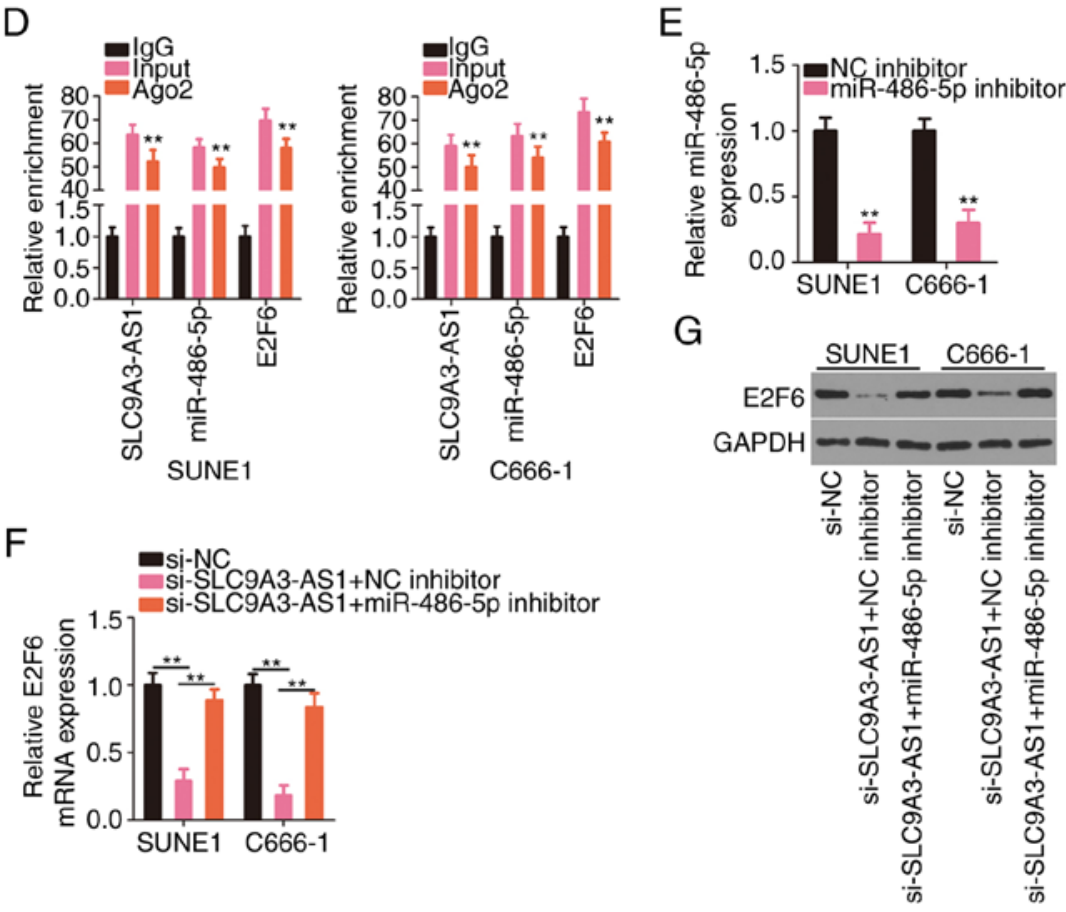

mSi-NC

Si-SLC9A3-AS1+NC inhibitor -si-SLC9A3-AS1+miR-486-5p inhibitor

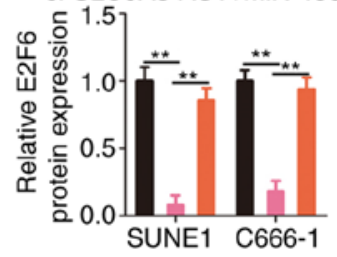

$\mathrm{F}$

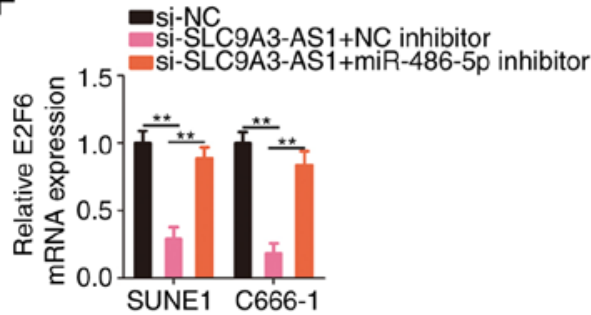

Figure 5. SLC9A3-AS1 upregulates E2F6 in NPC cells by sequestering miR-486-5p. (A and B) E2F6 levels were measured in NPC cells with SLC9A3-AS1 knockdown. (C) The positive relationship between SLC9A3-AS1 and E2F6 expression in NPC tissues. (D) The enrichment of SLC9A3-AS1, miR-486-5p, and E2F6 in the IgG, Ago2, and input groups was assessed with the RIP assay. (E) miR-486-5p level in NPC cells transfected with the miR-486-5p inhibitor or NC inhibitor was quantified using RT-qPCR. (F and G) E2F6 level was quantified in NPC cells co-transfected with si-SLC9A3-AS1 and miR-486-5p inhibitor or NC inhibitor. ${ }^{* *} \mathrm{P}<0.01$. SLC9A3-AS1, SLC9A3 antisense RNA 1; E2F6, E2F transcription factor 6; miR, microRNA; NPC, nasopharyngeal carcinoma; si-, small interfering; NC, negative control.

expression levels were observed in NPC cells (Fig. 5C). Notably, compared with the IgG group, the enrichment of SLC9A3-AS1, miR-486-5p, and E2F6 was considerably enhanced in the Ago2 group (Fig. 5D). Rescue experiments were implemented to assess whether miR-486-5p elicited the modulatory roles of SLC9A3-AS1 on E2F6. RT-qPCR verified the efficiency of the miR-486-5p inhibitor transfection and revealed that it considerably decreased miR-486-5p expression (Fig. 5E). Then, miR-486-5p inhibitor or NC inhibitor were transfected into SLC9A3-AS1 depleted-NPC cells, followed by the detection of E2F6 expression. Treatment of miR-486-5p inhibitor significantly restored the E2F6 (Fig. 5F and G) expression, which was previously reduced by SLC9A3-AS1 silencing. Therefore, SLC9A3-AS1 functioned as a ceRNA for miR-486-5p, thus upregulating E2F6 expression in NPC.

miR-486-5p downregulation or E2F6 overexpression attenuates the influences of si-SLC9A3-AS1 on the biological functions of NPC cells. Rescue experiments were actualized to ascertain whether the miR-486-5p/E2F6 axis was indispensable for the si-SLC9A3-AS1-mediated inhibition of malignancy in
NPC. The inhibition of miR-486-5p reversed the suppressive effect of si-SLC9A3-AS1 on NPC cell proliferation (Fig. 6A) and colony formation (Fig. 6B). In addition, NPC cell apoptosis was increased by the silencing of SLC9A3-AS1 but this effect was reversed after miR-486-5p inhibitor co-transfection (Fig. 6C). Similarly, the migration and invasion (Fig. 6D) of the si-SLC9A3-AS1-transfected NPC cells were impaired, but these changes were abolished upon miR-486-5p downregulation. The overexpression efficiency of pcDNA3.1-E2F6 was confirmed by western blotting (Fig. 7A). The inhibition of proliferation (Fig. 7B) and colony formation (Fig. 7C) of the cells with silenced SLC9A3-AS1 was restored by the transfection of pcDNA3.1-E2F6. Moreover, si-SLC9A3-AS1-induced NPC cell apoptosis was reversed by E2F6 overexpression (Fig. 7D). Furthermore, migration and invasion (Fig. 7E) were suppressed in NPC cells after SLC9A3-AS1 interference but recovered after the introduction of E2F6. In summary, SLC9A3-AS1 promoted carcinogenesis in NPC cells by targeting the miR-486-5p/E2F6.

Interference with SLC9A3-AS1 restrains NPC tumour growth in vivo. SUNE1 cells overexpressing sh-SLC9A3-AS1 were 

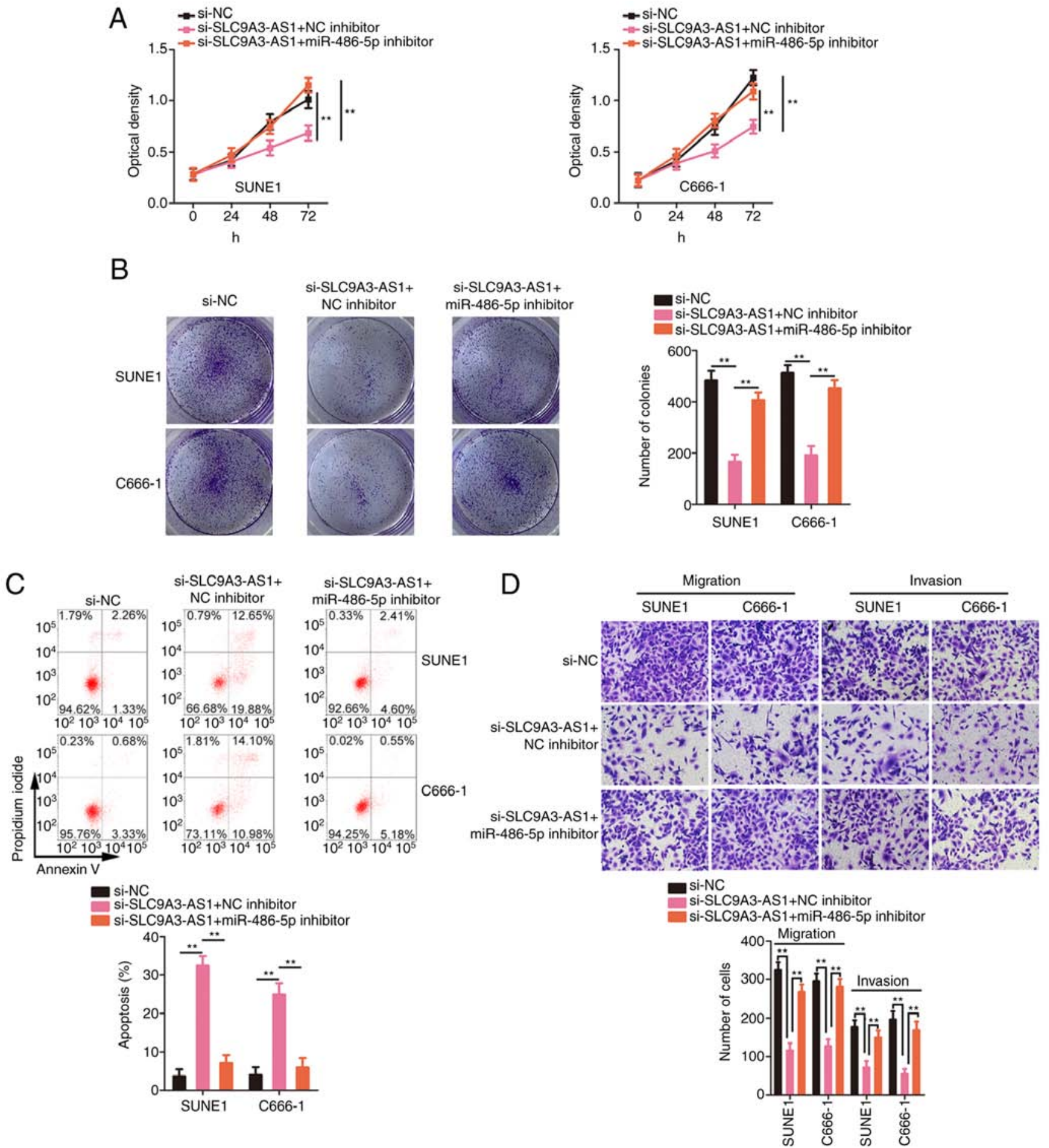

Figure 6. miR-486-5p inhibitor treatment abolishes the anti-oncogenic effects of si-SLC9A3-AS1 on NPC cells. (A and B) SLC9A3-AS1-silenced NPC cells were treated with the miR-486-5p inhibitor or NC inhibitor; their proliferative and colony-forming capacities were assayed. (C) Flow cytometric analysis was implemented for the apoptosis quantification in SLC9A3-AS1-silenced NPC cells which were further treated with miR-486-5p inhibitor or NC inhibitor. (D) The motility of NPC cells which received the aforementioned treatment. ${ }^{* *} \mathrm{P}<0.01$. miR, microRNA; si-, small interfering; SLC9A3-AS1, SLC9A3 antisense RNA 1; NPC, nasopharyngeal carcinoma; NC, negative control.

subcutaneously inoculated into nude mice to establish a mouse xenograft model. The xenograft tumours originating from sh-SLC9A3-AS1 were smaller in size (Fig. 8A) and volume (Fig. 8B) than sh-NC group. The tumours were also lighter in the sh-SLC9A3-AS1 group (Fig. 8C). In addition, SLC9A3-AS1, miR-486-5p, and E2F6 expression levels in xenograft tumours were analysed, revealing that SLC9A3-AS1 was expressed at a low level (Fig. 8D), whereas miR-486-5p was overexpressed (Fig. 8E) in the tumours injected with sh-SLC9A3-AS1-transfected cells. Moreover, E2F6 protein level was downregulated in the SLC9A3-AS1-silencing group (Fig. 8F). In addition, as demonstrated by immunohistochemistry, xenograft tumours in the sh-SLC9A3-AS1 group expressed E2F6 and Ki-67 at lower levels (Fig. 8G). These data confirmed that the depletion of SLC9A3-AS1 suppressed NPC tumour growth in vivo. 
A
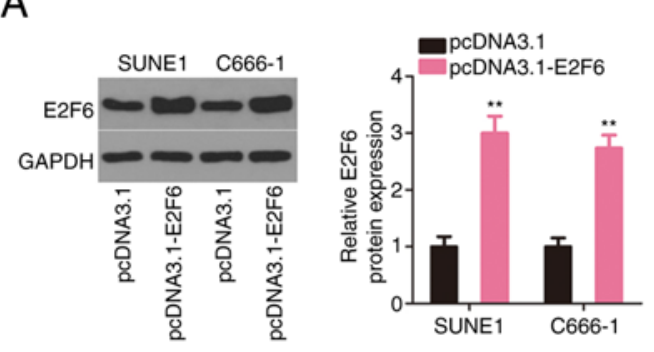

C
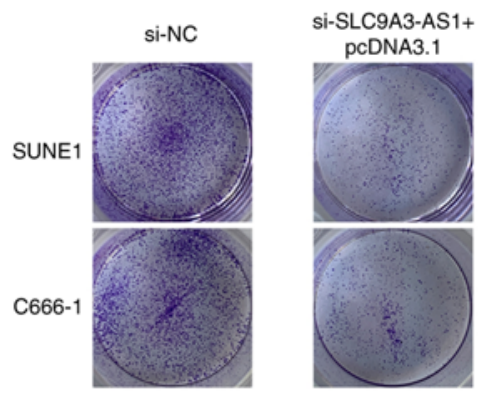

D

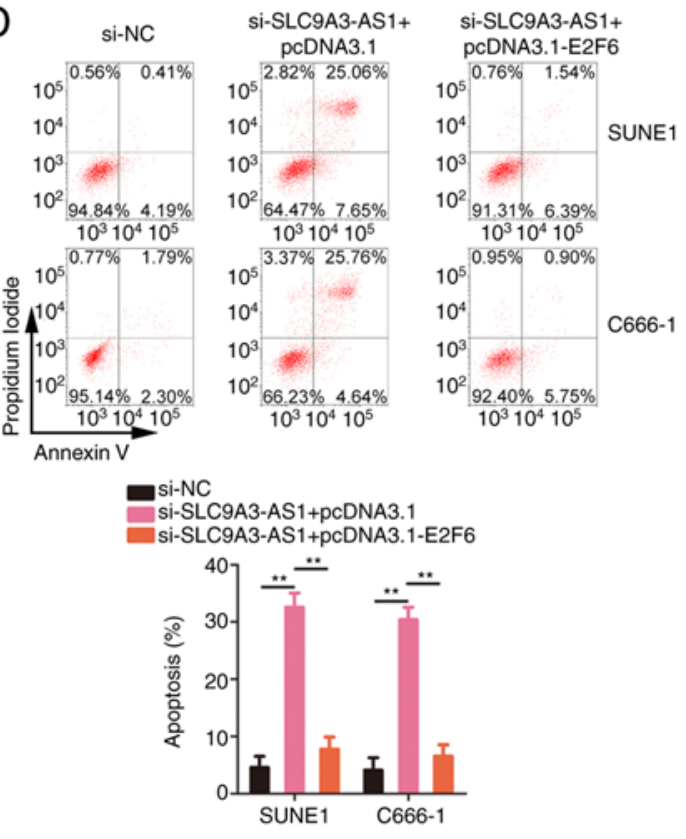

B

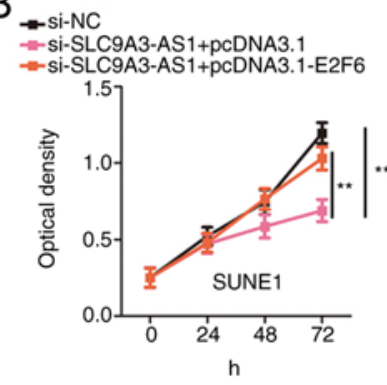

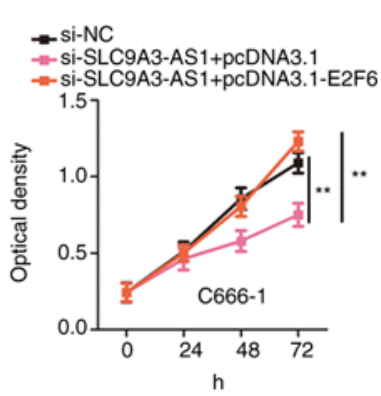

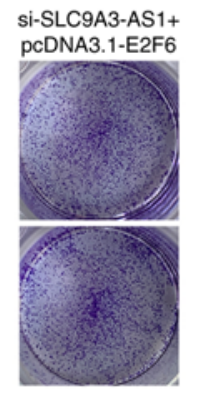

E
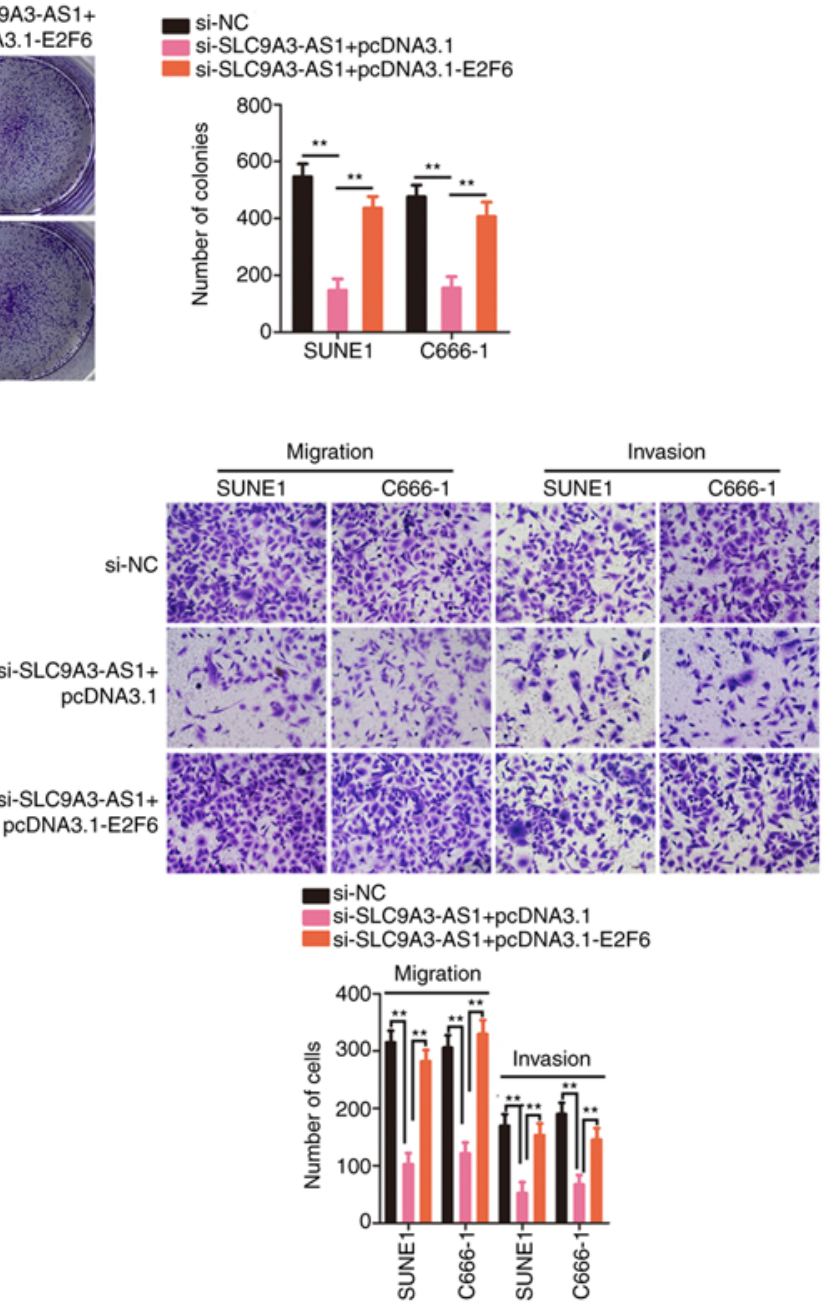

Figure 7. The inhibitory actions of si-SLC9A3-AS1 on NPC are offset by E2F6 overexpression. (A) The upregulation of E2F6 by pcDNA3.1-E2F6 was demonstrated. (B and C) SLC9A3-AS1-silenced NPC cells were transfected with pcDNA3.1-E2F6 or pcDNA3.1, and subjected to the determination of cell proliferation and colony formation. (D) The apoptosis of si-SLC9A3-AS1-transfected NPC cells underwent pcDNA3.1-E2F6 or pcDNA3.1 treatment was assessed using flow cytometry. (E) The motility of NPC cells which received the aforementioned treatment was detected. ${ }^{* *} \mathrm{P}<0.01$. si-, small interfering; SLC9A3-AS1, SLC9A3 antisense RNA 1; NPC, nasopharyngeal carcinoma; E2F6, E2F transcription factor 6; NC, negative control.

\section{Discussion}

Based on accumulating evidence, aberrantly expressed lncRNAs in tumours perform significant functions in oncogenesis and cancer progression (24-26). Extensive lncRNAs are aberrantly expressed in NPC and participate in the malignant processes of NPC (27-29). More than 50,000 lncRNAs are present in the human genome (30); however, most of them have not been investigated in the context of NPC and thus require further exploration. To the best of our knowledge, the present study is the first to analyse the expression and biological roles of SLC9A3-AS1 in NPC. The related mechanisms underlying the role played by SLC9A3-AS1 in NPC were also investigated.

SLC9A3-AS1 has been revealed as an overexpressed lncRNA and has been proposed as a biomarker for lung cancer (22). However, little is known about whether SLC9A3-AS1 regulates NPC. The present research revealed high SLC9A3-AS1 expression in NPC. Patients with NPC with a high level of SLC9A3-AS1 presented a shorter overall survival than patients with a low SLC9A3-AS1 level. 
A

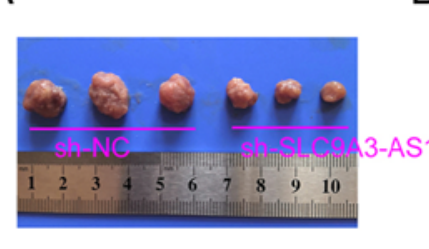

E

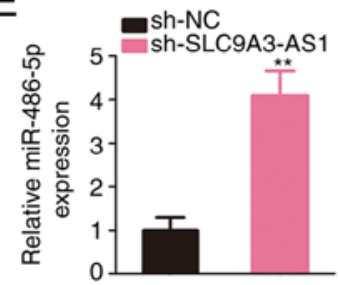

B

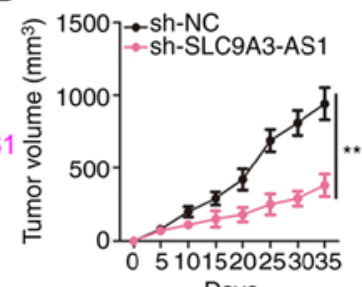

Days
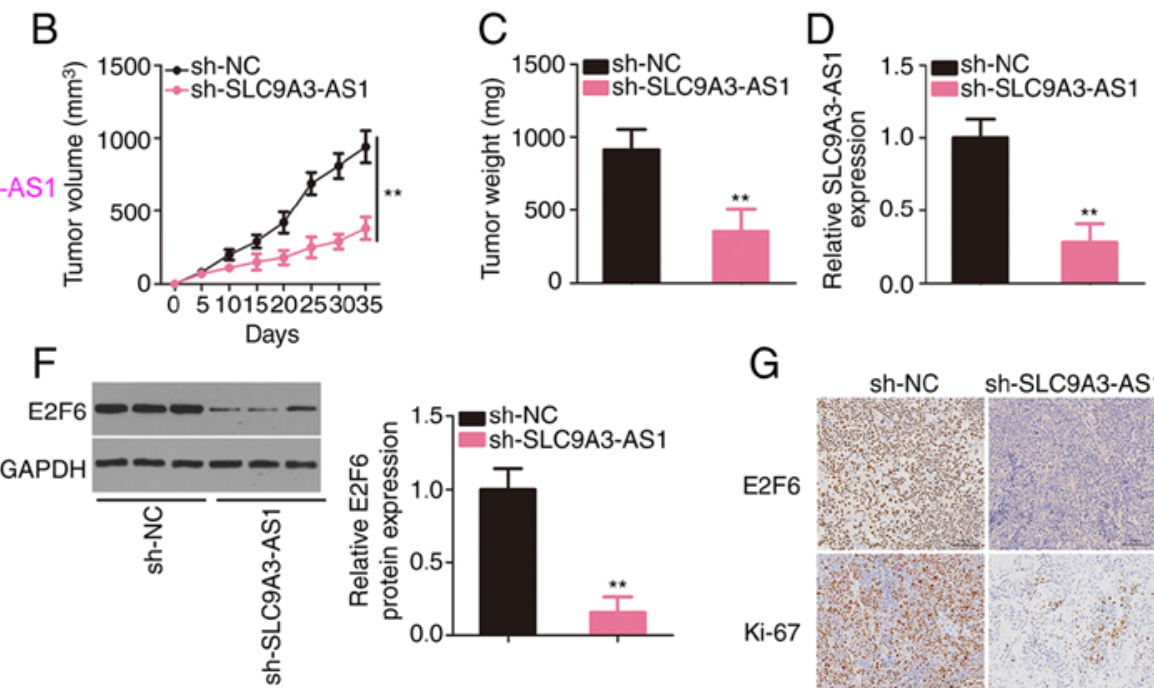

G

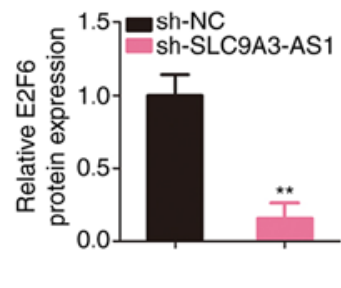

sh-NC sh-SLC9A3-AS1

E2F6

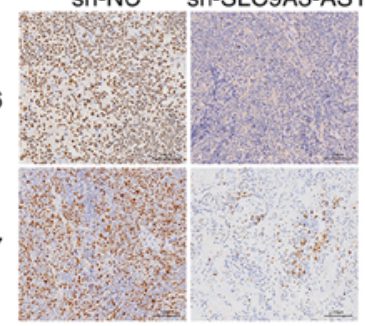

Figure 8. SLC9A3-AS1 silencing hinders tumour growth in vivo. (A) Xenograft tumours obtained from groups sh-SLC9A3-AS1 or sh-NC. (B) Tumour volume was measured with 5-day intervals and plotted to construct growth curves. (C) At the end of the experiment, tumours were collected and weighed. (D and E) SLC9A3-AS1 and miR-486-5p levels in the xenograft tumours were measured. (F) E2F6 protein level in xenograft tumours was detected via western blotting. (G) Immunohistochemical staining for E2F6 and Ki-67 in xenografts. ${ }^{* *}$ P<0.01. SLC9A3-AS1, SLC9A3 antisense RNA 1; sh-, short hairpin; $\mathrm{NC}$, negative control; miR, microRNA; E2F6, E2F transcription factor 6.

Functionally, loss of SLC9A3-AS1 produced anti-carcinogenic effects in NPC, and participated in the regulation of cell proliferation, colony formation, apoptosis, migration and invasion in vitro. Animal experiments further revealed that the interference of SLC9A3-AS1 hindered the growth of NPC tumours in vivo. Collectively, the aforementioned observations highlight SLC9A3-AS1 as an effective diagnostic biomarker and treatment target in NPC.

The localization of IncRNAs determines their mechanisms of action (31). LncRNAs that are primarily distributed in the cytoplasm function as miRNA sponges by directly interacting with miRNAs through miRNA response elements, subsequently weakening the inhibition of target genes by miRNAs (32). Numerous lncRNAs are reported to have roles in NPC as ceRNAs. For instance, a lncRNA called MEG3 facilitates NPC cell autophagy and apoptosis by working as a ceRNA for miR-21 and subsequently upregulating PTEN (33).

The molecules involved in the mechanisms of SLC9A3-AS1 in NPC which have remained largely ambiguous have been identified in the present study. Subcellular fractionation assays verified the theoretical basis for SLC9A3-AS1 as a ceRNA in the present study. According to bioinformatics predictions, SLC9A3-AS1 possessed complementary binding sequences for miR-486-5p. The direct target interaction between SLC9A3-AS1 and miR-486-5p in NPC was confirmed by applying luciferase reporter assay. A downstream target of miRNA is essential for the ceRNA network (34). Further mechanistic investigation revealed that E2F2 was directly targeted and negatively controlled by miR-486-5p. SLC9A3-AS1 was revealed to sponge miR-486-5p away from E2F6; thus, the silencing of SLC9A3-AS1 decreased E2F6 expression in NPC cells. Moreover, the RIP assay indicated that SLC9A3-AS1, miR-486-5p, and E2F6 all directly interacted with Ago2 in NPC cells, implying the coexistence of the three RNAs in an RNA-induced silencing complex. Furthermore, a positive expression relationship between SLC9A3-AS1 and E2F6 and an inverse relationship between E2F6 and miR-486-5p were observed in NPC tissues. In other words, the correlations among SLC9A3-AS1, miR-486-5p and E2F6 have allowed us to propose a new ceRNA pathway in NPC cells.

Notably, miR-486-5p is dysregulated in numerous human cancers. For example, miR-486-5p is highly expressed and has a carcinogenic role in non-small cell lung (35), prostate (36), and pancreatic (37) cancers. Conversely, miR-486-5p is expressed at low levels in osteosarcoma (38), colorectal cancer (39), and thyroid cancer (40) and is described to have an anti-oncogenic function. Therefore, miR-486-5p expression and function exhibit notable tissue specificity in human cancers. However, further studies are required to understand whether miR-486-5p contributes to the malignancy of NPC. Decreased miR-486-5p level was observed in NPC, and overexpressed miR-486-5p exerted tumour-inhibiting effects during NPC progression. Furthermore, E2F6, a member of the nuclear transcription factor E2F family, served as a crucial mediator of miR-486-5p action in NPC.E2F6 is regulated by multiple miRNAs in human cancer (41-43), and the present study identified a similar trend for E2F6 in NPC. Notably, the final rescue experiments revealed that the modulatory activities of SLC9A3-AS1 silencing on NPC cells could be reversed by miR-486-5p downregulation or E2F6 reintroduction. In summary, the miR-486-5p/E2F6 axis is a crucial downstream effector through which SLC9A3-AS1 exerted oncogenic regulation in NPC.

The phenotypic studies of NP69 compared to NPC cells with the manipulation of SLC9A3-AS1 expression could help to further understand the contribution of SLC9A3-AS1 during NPC progression. However, our study did not execute the phenotypic studies, and it was a limitation of our research, which will be addressed in the future.

The present study revealed that SLC9A3-AS1 exerted carcinogenic effects on NPC cells. SLC9A3-AS1 functioned as a ceRNA to sequester miR-486-5p, subsequently inducing E2F6 overexpression and regulating the 
malignant characteristics of NPC. Therefore, the newly identified SLC9A3-AS1/miR-486-5p/E2F6 axis may provide novel targets for therapeutic development in the future.

\section{Acknowledgements}

Not applicable.

\section{Funding}

The present study was supported by the People's Hospital of Rizhao.

\section{Availability of data and materials}

The datasets used and/or analysed during the present study are available from the corresponding author upon reasonable request.

\section{Authors' contributions}

JL and FZ conceived the research. JL, DL, XZ, CL and FZ conducted the experiments. JL and FZ drafted the manuscript. FZ acquired, analysed and interpreted the data. All authors read and approved the final manuscript.

\section{Ethics approval and consent to participate}

The present study was approved by the Ethics Committee of the People's Hospital of Rizhao (Rizhao, China). All participants provided written informed consent. All experiments involving animals were approved by the Animal Ethics Committee of the People's Hospital of Rizhao.

\section{Patient consent for publication}

Not applicable.

\section{Competing interests}

The authors declare that they have no competing interests.

\section{References}

1. Chua ML, Wee JT, Hui EP and Chan AT: Nasopharyngeal carcinoma. Lancet 387: 1012-1024, 2016.

2. Lee AW, Ng WT, Chan YH, Sze H, Chan C and Lam TH: The battle against nasopharyngeal cancer. Radiother Oncol 104: 272-278, 2012

3. Wei KR, Zheng RS, Zhang SW, Liang ZH, Ou ZX and Chen WQ: Nasopharyngeal carcinoma incidence and mortality in China in 2010. Chin J Cancer 33: 381-387, 2014.

4. Lee AW, Tung SY, Ngan RK, Chappell R, Chua DT, Lu TX, Siu L, Tan T, Chan LK, Ng WT, et al: Factors contributing to the efficacy of concurrent-adjuvant chemotherapy for locoregionally advanced nasopharyngeal carcinoma: Combined analyses of NPC-9901 and NPC-9902 Trials. Eur J Cancer 47: 656-666, 2011

5. Tan WL, Tan EH, Lim DW, Ng QS, Tan DS, Jain A and Ang MK: Advances in systemic treatment for nasopharyngeal carcinoma. Chin Clin Oncol 5: 21, 2016.

6. Tao Q and Chan AT: Nasopharyngeal carcinoma: Molecular pathogenesis and therapeutic developments. Expert Rev Mol Med 9: 1-24, 2007.

7. Lam WK and Chan JY: Recent advances in the management of nasopharyngeal carcinoma. F1000Res 7: F1000 Faculty Rev-1829, 2018.
8. Quinn JJ and Chang HY: Unique features of long non-coding RNA biogenesis and function. Nat Rev Genet 17: 47-62, 2016.

9. Peng WX, Koirala P and Mo YY: LncRNA-mediated regulation of cell signaling in cancer. Oncogene 36: 5661-5667, 2017.

10. Spizzo R, Almeida MI, Colombatti A and Calin GA: Long non-coding RNAs and cancer: A new frontier of translational research? Oncogene 31: 4577-4587, 2012.

11. Sanchez Calle A, Kawamura Y, Yamamoto Y, Takeshita F and Ochiya T: Emerging roles of long non-coding RNA in cancer. Cancer Sci 109: 2093-2100, 2018.

12. Liu $\mathrm{C}$, Zhang $\mathrm{H}$ and Liu $\mathrm{H}$ : Long noncoding RNA UCA1 accelerates nasopharyngeal carcinoma cell progression by modulating miR-124-3p/ITGB1 axis. Onco Targets Ther 12: 8455-8466, 2019.

13. Zhong Q, Chen Y and Chen Z: LncRNA MINCR regulates irradiation resistance in nasopharyngeal carcinoma cells via the microRNA-223/ZEB1 axis. Cell Cycle 19: 53-66, 2020.

14. Zhao CX, Zhu W, Ba ZQ, Xu HJ, Liu WD, Zhu B, Wang L, Song YJ, Yuan S and Ren CP: The regulatory network of nasopharyngeal carcinoma metastasis with a focus on EBV, lncRNAs and miRNAs. Am J Cancer Res 8: 2185-2209, 2018.

15. Guo H, Huang S, Li S, Yu H, Wu S and Zhou X: Prognostic significance of the long noncoding RNAs in nasopharyngeal carcinoma: A systematic review and meta-analysis. Cancer Manag Res 10: 1763-1779, 2018.

16. Liu H, Lei C, He Q, Pan Z, Xiao D and Tao Y: Nuclear functions of mammalian MicroRNAs in gene regulation, immunity and cancer. Mol Cancer 17: 64, 2018.

17. Ji W, Sun B and Su C: Targeting microRNAs in cancer gene therapy. Genes (Basel) 8: 21, 2017.

18. Tian Y, Tang L, Yi P, Pan Q, Han Y, Shi Y, Rao S, Tan S, Xia L, Lin J, et al: MiRNAs in radiotherapy resistance of nasopharyngeal carcinoma. J Cancer 11: 3976-3985, 2020.

19. Wang S, Claret FX and Wu W: MicroRNAs as therapeutic targets in nasopharyngeal carcinoma. Front Oncol 9: 756, 2019.

20. Lee KT, Tan JK, Lam AK and Gan SY: MicroRNAs serving as potential biomarkers and therapeutic targets in nasopharyngeal carcinoma: A critical review. Crit Rev Oncol Hematol 103: 1-9, 2016.

21. Salmena L, Poliseno L, Tay Y, Kats L and Pandolfi PP: A ceRNA hypothesis: The Rosetta Stone of a hidden RNA language? Cell 146: 353-358, 2011.

22. Bai Y, Qu Y, Wu Z, Ren Y, Cheng Z, Lu Y, Hu J, Lou J, Zhao J, Chen $\mathrm{C}$ and Mao H: Absolute quantification and analysis of extracellular vesicle lncRNAs from the peripheral blood of patients with lung cancer based on multi-colour fluorescence chip-based digital PCR. Biosens Bioelectron 142: 111523, 2019.

23. Livak KJ and Schmittgen TD: Analysis of relative gene expression data using real-time quantitative PCR and the 2(-Delta Delta C(T)) method. Methods 25: 402-408, 2001.

24. Ghafouri-Fard S, Shoorei H, Anamag FT and Taheri M: The role of non-coding RNAs in controlling cell cycle related proteins in cancer cells. Front Oncol 10: 608975, 2020.

25. Qian Y, Shi L and Luo Z: Long Non-coding RNAs in cancer: Implications for diagnosis, prognosis, and therapy. Front Med 7: 612393, 2020.

26. McCabe EM and Rasmussen TP: LncRNA involvement in cancer stem cell function and epithelial-mesenchymal transitions. Semin Cancer Biol: Dec 17, 2020 (Epub ahead of print).

27. Zhao J, Liu D, Yang H, Yu S and He H: Long noncoding RNAs in head and neck squamous cell carcinoma: Biological functions and mechanisms. Mol Biol Rep 47: 8075-8090, 2020.

28. $\mathrm{Wu} \mathrm{J}$ and Hann SS: Functions and roles of long-non-coding RNAs in human nasopharyngeal carcinoma. Cell Physiol Biochem 45: 1191-1204, 2018.

29. Dong Q, Zhou L, Liu F, Ao F, Gong X, Jiang C, Yuan Z and Li J: Long non-coding RNAs in the development, diagnosis and prognosis of nasopharyngeal carcinoma. Int J Clin Exp Pathol 10: 8098-8105, 2017.

30. Xu J, Bai J, Zhang X, Lv Y, Gong Y, Liu L, Zhao H, Yu F, Ping Y, Zhang G, et al: A comprehensive overview of IncRNA annotation resources. Brief Bioinform 18: 236-249, 2017.

31. Zhang K, Shi ZM, Chang YN, Hu ZM, Qi HX and Hong W: The ways of action of long non-coding RNAs in cytoplasm and nucleus. Gene 547: 1-9, 2014.

32. Rashid F, Shah A and Shan G: Long non-coding RNAs in the cytoplasm. Genomics Proteomics Bioinformatics 14: 73-80, 2016.

33. Lin L, Liu X and Lv B: Long non-coding RNA MEG3 promotes autophagy and apoptosis of nasopharyngeal carcinoma cells via PTEN up-regulation by binding to microRNA-21. J Cell Mol Med 25: 61-72, 2021. 
34. Sen R, Ghosal S, Das S, Balti S and Chakrabarti J: Competing endogenous RNA: The key to posttranscriptional regulation. ScientificWorldJournal 2014: 896206, 2014.

35. Gao ZJ, Yuan WD, Yuan JQ, Yuan K and Wang Y: miR-486-5p functions as an oncogene by targeting PTEN in non-small cell lung cancer. Pathol Res Pract 214: 700-705, 2018.

36. Yang Y, Ji C, Guo S, Su X, Zhao X, Zhang S, Liu G, Qiu X, Zhang Q, Guo $\mathrm{H}$ and Chen $\mathrm{H}$ : The miR-486-5p plays a causative role in prostate cancer through negative regulation of multiple tumor suppressor pathways. Oncotarget 8: 72835-72846, 2017.

37. Xia L, Song M, Sun M, Chen W and Yang C: miR-486 promotes capan-2 pancreatic cancer cell proliferation by targeting phosphatase and tensin homolog deleted on chromosome 10 (PTEN). Front Genet 10: 541, 2019.

38. He M, Wang G, Jiang L, Qiu C, Li B, Wang J and Fu Y: miR-486 suppresses the development of osteosarcoma by regulating PKC- $\delta$ pathway. Int J Oncol 50: 1590-1600, 2017.

39. Liu C, Li M, Hu Y, Shi N, Yu H, Liu H and Lian H: miR-486-5p attenuates tumor growth and lymphangiogenesis by targeting neuropilin-2 in colorectal carcinoma. Onco Targets Ther 9: 2865-2871, 2016.
40. Ma X, Wei J, Zhang L, Deng D, Liu L, Mei X, He X and Tian J: miR-486-5p inhibits cell growth of papillary thyroid carcinoma by targeting fibrillin-1. Biomed Pharmacother 80: 220-226, 2016.

41. An Y, Zhang J, Cheng X, Li B, Tian Y, Zhang X and Zhao F: miR-454 suppresses the proliferation and invasion of ovarian cancer by targeting E2F6. Cancer Cell Int 20: 237, 2020.

42. Cai Q, Zhao A, Ren LG, Chen J, Liao KS, Wang ZS and Zhang W: MiR-425 involves in the development and progression of renal cell carcinoma by inhibiting E2F6. Eur Rev Med Pharmacol Sci 22: 6300-6307, 2018.

43. Lu Z, Nian Z, Jingjing Z, Tao L and Quan L: MicroRNA-424/ E2F6 feedback loop modulates cell invasion, migration and EMT in endometrial carcinoma. Oncotarget 8: 114281-114291, 2017.

This work is licensed under a Creative Commons Attribution-NonCommercial-NoDerivatives 4.0 International (CC BY-NC-ND 4.0) License. 